\title{
Peritoneal Fluid Culture and Sensitivity in Case of Perforation Peritonitis: A Cross-Sectional Study
}

\section{Ohang Chaudhari ${ }^{1}$, Pranjal Shinde ${ }^{2}$, Honeypalsinh H Maharaul ${ }^{1}$}

Section: Healthcare

Sci. Journal Impact

Factor: $6.1(2018)$

ICV: 90.90 (2018)

(c) (3) (8)

Copyright@IJCRR

'Associate Professor, Department of Surgery, Dhiraj Hospital, Smt. BK Shah Medical Institute \& Research Centre, Sumandeep Vidyapeeth Deemed to be University, Piparia 391760, Gujarat, India; 'Resident, Department of Surgery, Dhiraj Hospital, Smt. BK Shah Medical lnstitute \& Research Centre, Sumandeep Vidyapeeth Deemed to be University, Piparia 39176o, Gujarat, India

\section{ABSTRACT}

Introduction: Peritonitis is a frequently encountered pathology by a general surgeon. The development of antimicrobial agents and the advancement of surgical techniques in the past decade have decreased morbidity and mortality. The complications range from minor wound infection to life-threatening septic shock and Systemic Inflammatory Response Syndrome (SIRS). Usage of appropriate antimicrobial agents that covers gram-positive, gram-negative, and anaerobic organisms provides good recovery in the patients. But inappropriate usage of antibiotics without following guidelines leads to the development of antibiotic resistance and treatment failure.

Objectives: To study the microbiological pattern of the peritoneal fluid in culture and sensitivity and to identify the pattern of antibiotic sensitivity for routinely used organisms.

Methods: This is cross-sectional study conducted in 50 patients with peritonitis and perforation confirmed on chest x-ray. Peritoneal fluid is isolated and nature of microorganism are characterized along with antibiotic sensitivity.

Results: In this study, the most common site of perforation leading to peritonitis is Duodenum followed by the stomach. Mostly secondary to peptic ulcer disease. The most common organism isolated was Klebsiella followed by E.coli, mixed cultures, Proteus, and pseudomonas. Most of the organisms isolated from the peritoneal fluid on culture were sensitive to the Cephalosporin group followed by Fluoroquinolones and macrolides.

Conclusion: Appropriate antibiotic use based on the sensitivity pattern is essential to improve the morbidity and mortality in patients who suffered from perforation secondary to peritonitis and to prevent antimicrobial resistance.

Key Words: Perforation, Peritonitis, Antimicrobials, Peritoneal fluid, Microorganism, Culture

\section{INTRODUCTION}

Peritonitis is a frequently encountered pathology by a general surgeon. It may be as simple as a simple duodenal perforation or a traumatic perforation of the bowel to a complex appendicular perforation or acute pancreatitis complicated with appendicular abscess, peritonitis remains the main culprit for mortality contributing up to $20 \%$ of mortality. Despite the availability of antimicrobial therapy, surgical techniques, and progress in intensive care support, management of peritonitis poses a complex challenge. ${ }^{1}$ The incidence and frequency of complications due to peritonitis in acute abdominal emergencies are different but the elderly, especially above 60 years and above, are more prone to complications. Due to the advances in the field of medicine, there is a sig- nificant increase in life expectancy. This led to an increase in the expectations of surgical care in the elderly which is an ever-increasing process. ${ }^{2,3}$

The prompt diagnosis followed by timely surgical exploration is a life-saving approach for saving life in case of secondary peritonitis. ${ }^{1}$ Surgical control of the peritonitis is the determinant factor for survival and has to be kept as o top priority in management. Even though control of sepsis requires state of the art intensive care facilities, the mortality rate proportionately increases with the time duration between the onset of hollow viscus perforation and the time of surgery. ${ }^{1}$

Peritonitis is associated with dangerous complications due to the high chance of contamination of the peritoneal cavity

\section{Corresponding Author:}

Honeypalsinh H Maharaul, Associate Professor, Department of Surgery, Dhiraj Hospital, Smt. BK Shah Medical Institute \& Research Centre, Sumandeep Vidyapeeth Deemed to be University, Piparia 391760, Gujarat, India; Ph: 9426345105; Email: honeypal.219@gmail.com

ISSN: 2231-2196 (Print)

Received: 22.07 .2020
ISSN: 0975-5241 (Online)

Revised: 27.09 .2020
Accepted: 05.11 .2020

Published: 14.12 .2020 
with organisms belonging to the Enterobacteriaceae familylike Escherichia coli, Klebsiella, Proteus, and enterococci. Either by direct action or via the secreted endotoxins they cause like threatening complications like Systemic Inflammatory Response Syndrome [SIRS]. Altheimer in 1930 is the first to isolate multiple organisms from the peritoneal fluid to explain the pathogenesis of the intra-abdominal sepsis. The evolution of the use of antibiotics over the past decade based on class 1 data had made the management of the sepsis feasible. To minimize the abscess formation, a decrease in the rate of morbidity and mortality combines antimicrobial approach has been considered. ${ }^{4,5}$ The current strategy towards the peritonitis includes correction of the underlying cause and use of broad-spectrum antibiotics to have better control of infection and preventing SIRS. ${ }^{5}$ A combined antimicrobial approach using broad-spectrum antibiotics that covers gram-positive, gram-negative, and anaerobes is effective in controlling sepsis. But the current problem is the development of resistance towards the commonly used antibiotics due to inappropriate use which is resulting in treatment failure. ${ }^{6-8}$

In this study, a detailed analysis of the peritoneal fluid from the patients suffering from peritonitis secondary to hollow viscous perforation was done including organisms identified and the pattern of the sensitivity was done. Based on this we were able to initiate appropriate antibiotics at the earliest which improves the outcome of the patients.

\section{MATERIALS AND METHODS}

Study Design: Cross-sectional study

Sample Size: 50

\section{Inclusion Criteria:}

Adults ( $>18$ years) presenting with clinical features of peritonitis and perforation confirmed on chest $\mathrm{x}$-ray

\section{Exclusion Criteria:}

1. Cases of primary peritonitis

2. Cases of peritonitis secondary to trauma

\section{Procedure}

Pre-operative Evaluation: Patients presenting to the emergency unit at Dhiraj hospital with features of peritonitis were admitted. After a detailed history and physical examination, an x-ray chest pa view was taken. If there is evidence of pneumo-peritoneum on $\mathrm{x}$-ray and if the patient fits the inclusion and exclusion criteria patient is included in the study.

Pre-operative Preparation: All routine investigations were done. An electrocardiogram is performed in all cases and an echocardiogram is done in patients with comorbidities. They were resuscitated with intravenous fluids and once the vitals stabilized after taking due consent from the patient and his/ her relatives an emergency laparotomy was performed.
Intra-operative Procedure: During the surgery, a midline incision was used for intra-abdominal access and peritoneal fluid was sent for microbial culture and sensitivity. After identifying the perforation depending on the size of the perforation and the status of the surrounding tissue live omental patch repair or resection anastomosis with or without diversion was performed followed by closure and abdomen after thorough lavage.

Post-operative Care: After surgery routine postoperative care with intravenous fluid, analgesic, antacids, and antibiotics were given. Initially, the patient was started on Inj. Cefotaxime and inj. Metronidazole. Using a Kirby-Bauer disc diffusion method using ampicillin, amikacin, ciprofloxacin, ceftriaxone, and cotrimoxazole and the culture reports were obtained. Antibiotics were started according to the sensitivity pattern of the organism grown in the culture.

\section{RESULTS AND DISCUSSION}

\section{Age distribution}

This study shows that the most common age group of presentation is about 31 to 40 years ( $38 \%$ ) followed by 21 to 30 years $(24 \%)$. The mean age of presentation is being 36.23 years. It is comparable to previous study. ${ }^{9}$ Middle age group is affected which may be attributed to food habits, lifestyle, working stress (Figure 1).

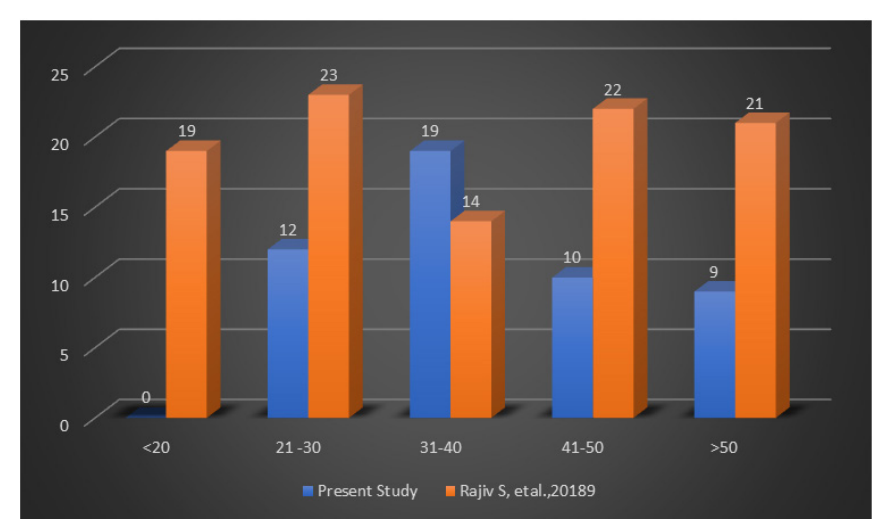

Figure 1: Comparison of age distribution found in the present study vs a study by Srivastava and Singh, $2018 .{ }^{9}$

\section{Gender distribution}

In our study (Table 1), the male to female ratio of the patients suffering from peritonitis secondary to hollow viscus perforation is 7.3:1 which is similar to Srivastava and Singh, 2018. ${ }^{9}$ This suggests male predominance which may be attributed to smoking, spicy food intake, alcohol intake low water intake in these population. 
Table 1: Gender Distribution

\begin{tabular}{lcc} 
SEX & Present Study & Srivastava and Singh, 20189 \\
Male & 44 & 75 \\
Female & 6 & 25 \\
\hline
\end{tabular}

\section{Effect on duration of Symptoms}

This study shows that most of the cases presented to us with symptoms of perforation after 2 to 3 days which is $46 \%$ followed by 4 to 5 days which is about $36 \%$. The mean duration of symptoms was about 2.66 days (Figure 2). This finding is less when compared to a mean of 6.2 days in a study conducted by Mutiibwa et al., 2013. ${ }^{4}$

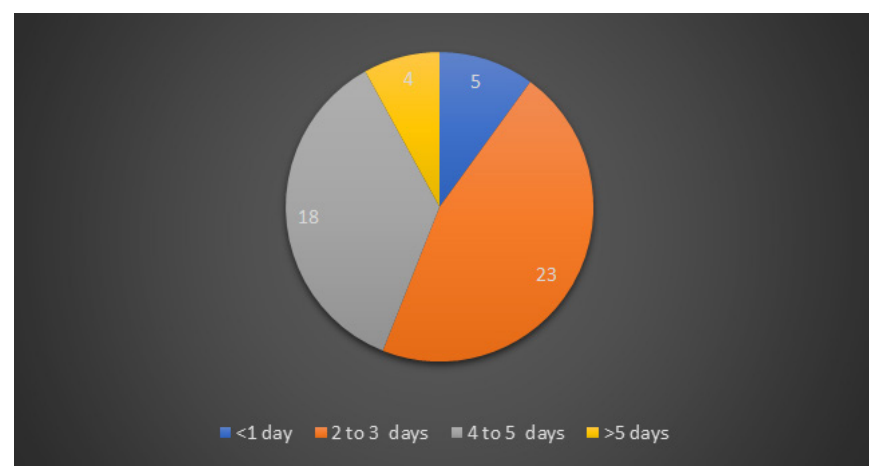

Figure 2: Pie diagram showing the distribution of the duration of the symptoms.

\section{Site of Perforation}

In our study, the most common site of perforation is Duodenal which is $42 \%$ followed by Gastric which is $34 \%$. The incidence of ileal, jejunal, and colonic perforation is $14 \%$, $4 \%$ and $6 \%$ respectively which is comparable to Rajiv S, et al., 2018 study (Figure 3). ${ }^{9}$ Duodenal and gastric perforation occurs due to food habits and alcohol intake which is common in India being a developing country. Jejunal perforation is rare and occurs due to blunt abdominal trauma during road traffic accidents. Ileal perforation is secondary to typhoid or tuberculosis which are common in India. ${ }^{8,9}$

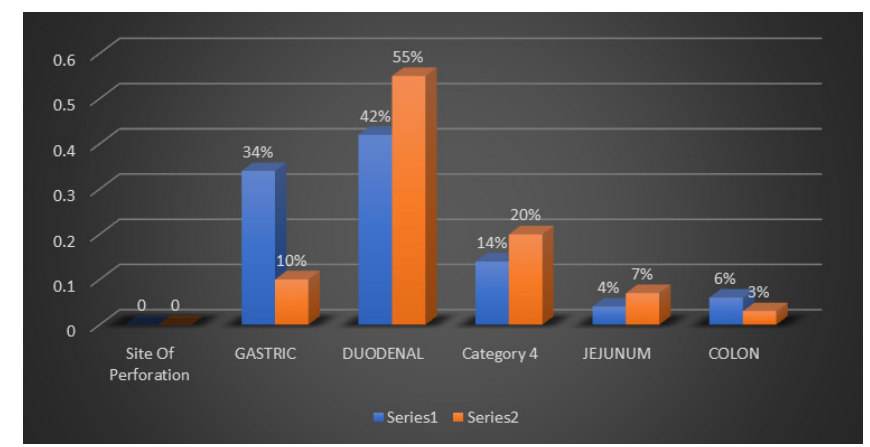

Figure 3: Graph showing the distribution of the site of Perforations.

\section{Cultured Organisms}

In this study, out of 50 cases (Table 2), the most common organism grown is Klebsiella species (46\%) followed by Escherichia coli (34\%) and no growth in about $14 \%$. In three cases of perforation, there was a growth of proteus, pseudomonas, and a combination of E Coli and Klebsiella in their culture. The above results were more or less similar to the study conducted by Mutiibwa et al., $2013^{4}$ on aerobic bacterial causes of secondary peritonitis and their antibiotic sensitivity pattern which showed, Klebsiella species being $37.9 \%$ and Escherichia coli $26.4 \%$ and no growth $13.8 \%$. Rajiv et al., $2018^{9}$ study Klebsiella species was identified in $52 \%$ while Escherichia coli in $36 \%$ and combination of both in $5 \%$.

Table 2: Frequency of the isolated organisms in the peritoneal fluid culture

\begin{tabular}{lccc} 
Organisms & $\begin{array}{c}\text { Present } \\
\text { Study }\end{array}$ & $\begin{array}{c}\text { Mutiibwa et al., } \\
\mathbf{2 0 1 3 ^ { 4 }}\end{array}$ & $\begin{array}{c}\text { Rajiv et al., } \\
\mathbf{2 0 1 8}\end{array}$ \\
Ecoli & $34 \%$ & $26.4 \%$ & $36 \%$ \\
Klebsiella & $46 \%$ & $37.9 \%$ & $52 \%$ \\
Klebsiella +Ecoli & $2 \%$ & - & $5 \%$ \\
Proteus & $2 \%$ & - & $2 \%$ \\
Pseudomonas & $2 \%$ & - & $2 \%$ \\
No growth & $14 \%$ & $13.8 \%$ & - \\
\hline
\end{tabular}

\section{Organism Cultured According to the Site of Perforations}

This study (Table 3) shows that the most common organism grown in gastric perforation was Klebsiella followed by $E$ coli. In the case of duodenal ulcer perforation $E$ coli being the most common 9 cases followed by Klebsiella in 7 cases, no growth in 3 cases and proteus in 1 case, and mixed growth in 1 case. In the case of ileal perforation, the common species being Klebsiella followed by pseudomonas. Most of the perforation presented to us after 2 to 3 days.

Table 3: Distribution of the organisms isolated from the peritoneal fluid culture

\begin{tabular}{lccccc} 
Perforations & Gastric & Duodenal & Ileal Jejunal Colonic \\
E.coli & 5 & 9 & 3 & 1 & 1 \\
Klebsiella & 10 & 7 & 1 & 1 & 1 \\
Proteus & & 1 & & & \\
Pseudomonas & & 1 & 2 & 1 & \\
Mixed & 2 & 3 & 1 & & 1 \\
Nogrowth & 17 & 21 & 7 & 2 & 3 \\
\hline
\end{tabular}




\section{Correlation of Ogranisms and Day of Perfora- tions}

This study (Table 4) shows that the most common organism grown during day 2 to 3 of perforation was E.coli (9 cases) followed by Klebsiella (7 cases). And most common organism during day 4 to 5 of perforation was also Klebsiella (10 cases) followed by Escherichia coli (9 cases). On day 1 of perforation, most of the cultures were negative for growth. In the case of perforation, with 5 days old at presentation most had Klebsiella species in their culture followed by mixed infection. Overall the most common organism being Klebsiella ( 20 cases) followed by E.coli (18 cases) in culture growth in perforation cases.

Table 4: Comparison of the Organisms Isolated with the Duration of Symptoms before Surgery

\begin{tabular}{lcccc} 
& Day $\mathbf{1}$ & Day2-3 & Day4-5 & >5days \\
E.coli & - & 9 & 9 & - \\
Klebsiella & - & 7 & 10 & 3 \\
No growth & 5 & 2 & - & - \\
Proteus & - & - & 1 & - \\
Pseudomonas & - & 1 & 2 & - \\
mixed & - & - & - & 1 \\
\hline
\end{tabular}

\section{Sensitivity Pattern for Common Antibiotics}

In our study (Table 5), E.coli showed sensitivity to Ceftriaxone in $77.8 \%$ of cases, Ciprofloxacin, and amikacin in $72.2 \%$ of cases. For klebsiella, ceftriaxone being more sensitive than $95 \%$ followed by amikacin $75 \%$ and ciprofloxacin $70 \%$. Proteus and pseudomonas species which are less commonly found in the peritoneal cavity in case of perforation peritonitis are sensitive to ceftriaxone and ciprofloxacin. But proteus was resistant to amikacin.

Table 5: Distribution of the Sensitivity of the Organisms Isolated on Peritoneal Fluid Culture and Sensitivity

\begin{tabular}{lcccc} 
& $\begin{array}{c}\text { E.Coli } \\
\mathbf{N}=\mathbf{1 8}\end{array}$ & $\begin{array}{c}\text { Klebsiella } \\
\mathbf{N}=\mathbf{2 0}\end{array}$ & $\begin{array}{c}\text { Proteus } \\
\mathbf{N}=\mathbf{1}\end{array}$ & $\begin{array}{c}\text { Pseu- } \\
\text { domonas } \\
\mathbf{N}=3\end{array}$ \\
Ampicillin & 2 & 1 & - & - \\
Ciproflox & 13 & 14 & 1 & 1 \\
Ceftriaxone & 14 & 19 & 1 & 1 \\
Cotrimoxazole & 1 & 2 & - & 1 \\
Amikacin & 13 & 15 & - & 1 \\
\hline
\end{tabular}

\section{Sensitivity Pattern for E. Coli}

This chart (Figure 4) shows the sensitivity pattern of $E$ coli, which shows that the most common antibiotic sensitive is ceftriaxone followed by ciprofloxacin and amikacin. And is resistant to ampicillin and co-trimoxazole in most cases. There has been a mild increase in resistance to ciprofloxacin and amikacin.

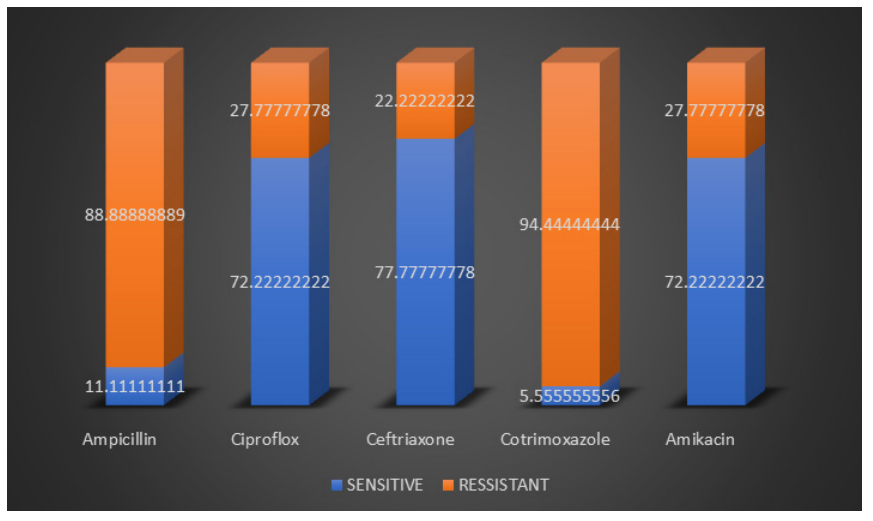

Figure 4: Sensitivity Pattern for E. Coli.

\section{Sensitivity Pattern for Klabsiella}

This chart (Figure 5) shows the sensitivity and resistant pattern of Klebsiella which shows that these species were susceptible to the ceftriaxone group of drugs in most of the cases followed by amikacin and ciprofloxacin in the order. The organisms showed resistance to Ampicillin and cotrimoxazole in most of the cases. In this study, the most common site of perforation leading to peritonitis is Duodenum followed by the stomach. Mostly secondary to peptic ulcer disease. The most common organism isolated was Klebsiella followed by E.coli, mixed cultures, proteus, and pseudomonas. Most of the organisms isolated from the peritoneal fluid on culture were sensitive to the Cephalosporin group followed by Fluoroquinolones and macrolides.

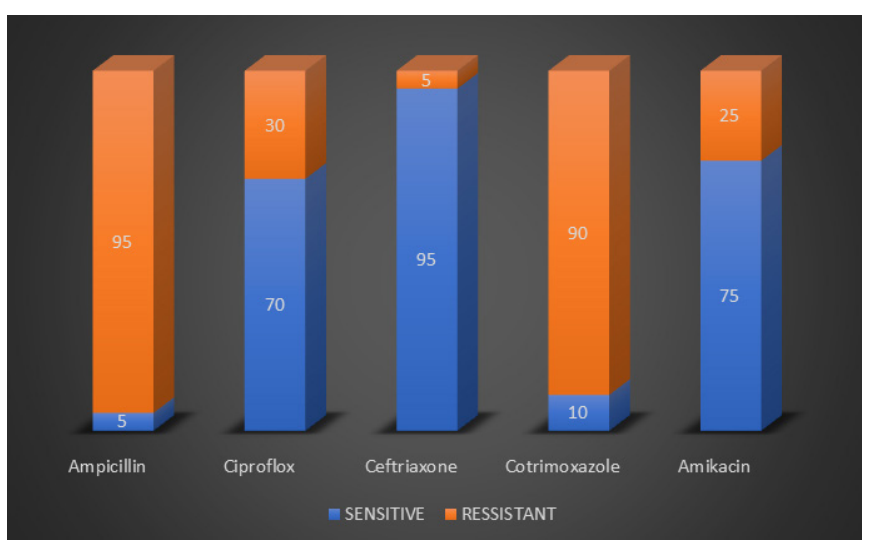

Figure 5: Sensitivity Pattern for Klabsiella.

\section{CONCLUSION}

Appropriate antibiotic use based on the sensitivity pattern is essential to improve the morbidity and mortality in patients who suffered from perforation secondary to peritonitis and to prevent antimicrobial resistance. 


\section{ACKNOWLEDGEMENT}

We are Thankful to our mentor Dr. Vijoy Singh who has contributed to making this study possible.

\section{Conflict of Interest: NIL}

\section{Source of Funding: NIL}

\section{REFERENCES}

1. Strobel O, Werner J, Büchler MW. Surgical therapy of peritonitis. Chirurg. 2011;82(3):242-8.

2. Nishida K, Ohinaga K, Miyazawa Y. Emergency abdominal surgery in patients aged 80 years and older. Surg Today 2000; 30:22-27.
3. Rigberg D, Cole M, Hiyama D, McFadden D. Surgery in nineties. Am Surg 2000; 66: 813-6.

4. Mutiibwa D, Tumusiime G. Aerobic bacterial causes of secondary peritonitis and their sensitivity pattern in non-traumatic small bowel perforation. East cent Afr J Surg 2013; 18(2):34-39.

5. Weinstein RA. Controlling antimicrobial resistance in hospitals: infection control and use of antibiotics. Emerg Infect Dis. 2001; 7(2):188-192.

6. Maingot's Abdominal Surgery, 10 $0^{\text {th }}$ Edition. 2012: 633-650.

7. Sabiston Textbook of Surgery, $19^{\text {th }}$ Edition, 2017: 1088-1114.

8. Schwartz's Principle of Surgery, 10th Edition, 2019:1035-1099.

9. Srivastava R, Singh RK. Clinical evaluation of the patient with perforation peritonitis and their peritoneal fluid analysis for culture and sensitivity. Int Surg J 2018;5(6):2299-2303. 